\title{
Human Biomonitoring of Inorganic Elements at the UK Health and Safety Laboratory
}

\author{
J. Morton ${ }^{1}$, E. Leese, E. Tan and J. Cocker \\ ${ }^{1}$ Health \& Safety Laboratory Harpur Hill, Buxton, SK17 9JN, UK, jackie.morton@hsl.gov.uk
}

\begin{abstract}
Background levels of 38 elements of a UK population are presented here from 137 people. It has been possible to assign $95^{\text {th }}$ percentile data most of these elements. This presentation will summarise the findings of the background level study and show how this compares with biological monitoring data collected from routine occupational monitoring.
\end{abstract}

Key words: Biological monitoring, background levels, urine, exposure, metals.

\section{Introduction}

Biological samples such as urine and blood provide matrices where endogenous status of metals can be determined. These measurements aid the assessment of systemic exposure to metals in both workers and nonexposed persons. Determining background levels of metals in urine samples from non-exposed people allows for normal levels in a population to be established. This provides a baseline for a direct comparison (and interpretation) of biological monitoring (BM) results from workers exposed to metals.

Exposure to rare metals is becoming a concern because of their increasing use in many new areas of technology. The development of technologies such as solar panels and semiconductors (and the subsequent recycling of all of these components) mean that there is the potential for people in the UK to be exposed to rare metals. In order to interpret exposure to these metals it is first necessary to establish current background levels of metals in urine samples from unexposed people.

In this project urine sample were collected from unexposed persons and the samples were analysed by ICP-MS for a wide range of metals including rare and unusual metals.

\section{Methods and Materials}

Urine samples $(\mathrm{n}=297)$ have been collected from 137 volunteers who had not been occupationally exposed to metals. All participating individuals provided informed consent, in accordance with $\mathrm{HS}(\mathrm{G}) 167^{1}$. Each of the samples has been analysed for 38 elements and for creatinine.

Urine samples were stored at $-20^{\circ} \mathrm{C}$ before analysis. Creatinine measurements were measured by alkaline picrate method on a COBAS- MIRA spectrophotometer (Roche Diagnostic Systems, Basel, Switzerland). Urine samples were prepared and analysed in different diluents and ICP-MS methods depending on the elements. Analysis was undertaken on an X7 Series 2 ICP-MS Thermo Fisher Scientific (Hemel Hempstead, UK). Urine samples were analysed for different elements by four methods: in normal mode diluted 20 -fold with $1 \%$ nitric acid; in collision-cell technology (CCT) mode with $3.5 \mathrm{ml} / \mathrm{min} 7 \%$ helium in hydrogen diluted 20 -fold with $1 \%$ nitric acid; in normal mode diluted 10 -fold with $1 \%$ $\mathrm{HCl}$; and for mercury alone in normal mode diluted 10 fold with $1 \%$ nitric acid.

External quality control (EQC) consisted of ClinChek Urine controls (Recipe Chemicals, Munich, Germany). Internal quality controls were made for elements for which no EQC existed.

Mixed effects models were fitted within a Bayesian framework that explicitly modelled the left censored observations and inference was made using Markov Chain Monte Carlo methods, implemented in the WinBUGs software.

\section{Results and Discussion}

The non-occupationally exposed group generated 297 urine samples collected from 137 non-occupationally exposed people (85 males and 52 females). The limits of quantification (calculated by LOD $(3 \mathrm{xSD}$ of blank) 
multiplied by the dilution factor) and the quality control data are established for each element.

Summary statistics, including 95th percentile reference levels were established for each element. These results are reported both creatinine corrected and uncorrected for creatinine. Mixed effect analysis was carried out on the elements, however, based on the high percentage of results $<\mathrm{LOQ}$, the analysis was not carried out for the following elements: $\mathrm{Sb}, \mathrm{Pb}, \mathrm{Bi}, \mathrm{Nb}, \mathrm{Ag}, \mathrm{Os}$, $\mathrm{Pt}, \mathrm{Cr}, \mathrm{Mn}$, and $\mathrm{Sn}$. The mixed effect analysis showed that, for all of the remaining elements, creatinine correcting the data in all cases gave a reduction in variability or no significant difference in variability. No smoking effect was observed for any element that was modelled. Gender effects were observed for $\mathrm{Li}, \mathrm{B}, \mathrm{Co}$, $\mathrm{Cu}, \mathrm{Rb}, \mathrm{Sr}, \mathrm{Cs}, \mathrm{Hg}, \mathrm{Tl}, \mathrm{Te}, \mathrm{V}, \mathrm{Se}$ and W.

\section{Conclusions}

Reference levels for a wide range of elements have been established for a UK population. More elements are yet to be determined in this project. From the data presented here it would suggest that elemental concentrations in urine should be creatinine corrected. This data also shows that there are no major changes in the unexposed levels of 'routine' metals used in everyday biological monitoring at HSL.

\section{References}

HSE (1997) HSG 167 Biological Monitoring in the workplace http://www.hse.gov.uk/pubns/books /hsg167.htm 